\title{
A Study on Introduction of Intermediary for e-Collaboration between SMEs
}

\author{
Jemin Yang \\ Inha University \\ E-Mail: itip@inhaian.net \\ Ji-woong Shin \\ Inha University \\ E-Mail: inbody@inhaian.net \\ Young-wook Park \\ Inha University \\ E-Mail: global21@dreamwiz.com \\ Jaechon Park \\ Inha University \\ E-Mail: jcpack@inha.ac.kr
}

\begin{abstract}
Latest e-business trends are collaborations as alternatives to extreme competition and strategies for cost-saving and growth. But e-collaboration among SMEs(Small and Medium Size Enterprises) has too many constraints. Because SMEs are feeble in utilizing IT technology and lack driving force and concentration for informatization. In this context, we propose to introduce e-collaboration intermediary which can promote ecollaboration among SMEs. It is in charge of supporting that SMEs with the different system each other can form the collaborative business relationship while they use their own unique systems. We also show e-collaboration intermediary's system architecture which can be adoptable. Lastly, we think about expected effect in technical and business aspects. In the technical aspect, its application can be justified in its open, horizontal, and result-oriented characteristics. And in the business aspect, its application can be justified
\end{abstract}


in making the business activities efficient or creating the new business.

Keywords: E-Collaboration Intermediary, E-Collaboration Platform, Horizontal Collaboration

\section{INTERDUCTION}

Even though the e-business environment is generally considered as an opportunity to enhance the status of SMEs(small and medium enterprises), it still has a structure subordinate to large enterprises in reality; which means that SMEs lack understanding and utilizing the information and communication technologies which are treated importantly in the e-business.

SMEs can make a stepping-stone for the application of the e-business by using the driving force or concentration of large enterprises. But as long as the relationship limitation caused by the difference in the information level between SMEs and large enterprises exists, it is inevitable for the subordination of SMEs to be continued. Furthermore, collaboration in which large enterprises do not participate, namely, the collaboration of SMEs only, has more restrictions. It is because the collaboration of SMEs only has the limitation to establish the business model or process for the collaboration owing to the lack of even the driving force or concentration of large enterprises and SMEs are in low-level of informatizaton.

In this context, this paper aims at defining e-Collaboration Intermediary and suggesting its system architecture, which can promote the collaboration without large enterprises. The e-Collaboration Intermediary will take the role of supporting the collaboration in between SMEs by connecting the business models and processes each other of SMEs which require the collaboration. In addition, as it is necessary not only to establish the technological environment, but to estimate the business in the technological environment in order to see if the e-business collaboration can be set up actually or not, this paper aims at studying on the expected effect in both technology and business of the intermediary system architecture. 


\section{DEFINITION AND DESIRABLE STRUCTURE OF E-COLLABORATION}

\section{Definition of e-Collaboration}

The internet can enlarge the total size of market by lowering the entry barrier to the industry and promoting the competition and substitute goods, and largely increase the productivity of the enterprises as being applied to the business strategies. These benefits are not only for large enterprise, SMEs can also benefit from internet(Konings \& Roodhooft, 2002; Porter, 2001). Accordingly, not only large enterprise but SMEs intend to combine traditional enterprise environments with the concept of e-business(Steinfield, 2002).

Moreover, as it is the trend of the e-business to focus on the collaboration, it is because the e-Collaboration enables the enterprises to collaborate with more companies in less cost than in the existing off-line method, and the enterprises can more easily take the opportunity for the development by concentrating on the core capability of each through the collaboration. Apart from the essential purposes of enterprises such as the reduction of cost or focusing on development, the collaboration is considered important to overcome 'the uncertainty of environment or partnership' as the alternative for the strategy in the extreme competition(Watson, 2004).

From this point of view, the e-Collaboration can be defined to carry out the value creation of the enterprises through the adjustment and cooperation of each department or the various functions in the organizations by utilizing the technologies based upon the internet; the range of collaboration has been enlarged by real time sharing the information with internal department as well as the competitors (Anthony, 2000).

As the enterprises carry out the business activities by setting up each system, how to associate and realize the different data and process, namely, the technological consideration to establish the collaboration system is important. It is because the success or failure of e-business is up to how to use the information and communication technologies and whether to actually realize it as a system(Park \& Yang, 2005; Salo \& Karjaluoto, 2006). Therefore, to set up the system architecture is the important work for the realization of e-Collaboration.

\section{Desirable e-Collaboration structure}

Compared to the traditional business, as the e-business has the features to fortify and emphasize the information and distribution rather than the existing economic structure, 
and accordingly, the business relationship is treated especially important in the ebusiness(Sawhney \& Mandal, 2000). Nøkkentved \& Hedaa(2001) divided the relationship with the partners in the e-business into three such as Transactional Relationship, Information-Sharing Relationship, Collaborative Relationship.

Transactional Relationship is the stage of selling and buying goods each other, and the Information-Sharing Relationship is the stage to be evolved from the Transactional Relationship and in the level to exactly collect the information on the partners. The Collaborative Relationship means to carry out the equal tasks by setting the same purposes. Given that they only defined 'equal tasks and same purposes' as collaboration, we can get the ultimate direction in the e-business environment; horizontal work is reasonable. In addition, as Choi et al.(2005) explained that the relationship in between enterprises in the digital environment was to be decided by the dominating relation and ownership of shares, he pointed out especially that the lower is the level of domination, the more horizontal the collaboration gets, and can realize the balanced industrial organization. Considering the studies above, it is desirable that the relationship in between enterprises which participate in the collaboration should be the horizontal form with the power balanced each other.

On the other hand, Though the development of ICT (Information and Communication Technologies), which cause the spread of the e-business, makes the enterprises deal with the use of ICT as one of the important business strategies, in reality not all enterprises can utilize developed ICT. As they lack the skilled manpower and technological resources compared to large enterprises, SMEs has difficulty in setting up the information-oriented environment, and also takes longer time than large enterprises(Nie, 2007).

Of course, because of the recent decrease in cost for using ICT and the spread of recognition of needs to its adaptation, SMEs emphasize the strategies for utilizing ICT outsourcing(Chen et al., 2003; Teng et al., 1995). But SMEs are restricted in using ICT for the e-business owing to the professionalism and particularity of the e-business. Especially, considering the e-Collaboration as the most evolved form in the e-business model, SMEs are in the bigger difficulty(Pratt,2000; Rao et al., 2003).

Like this, premising the environment of SMEs in which they should be limited to using ICT through outsourcing besides the low-level of informatization compared to large enterprises, the introduction of e-Collaboration Intermediary as the third party can be considered as the solution to promote the e-Collaboration of SMEs only, which can be 
well supported by difference of information-oriented environment between large enterprises and SMEs; it is difficult to simply apply system of large enterprises to SMEs(Bernroider \& Koch,2000).

As shown in the above, considering the horizontal collaboration as more desirable, it is positive to propose the intermediary which can objectively adjust the balance of power of SMEs which participate in the collaboration. From this perspective, our paper suggests the most desirable e-Collaboration structure in between SMEs.

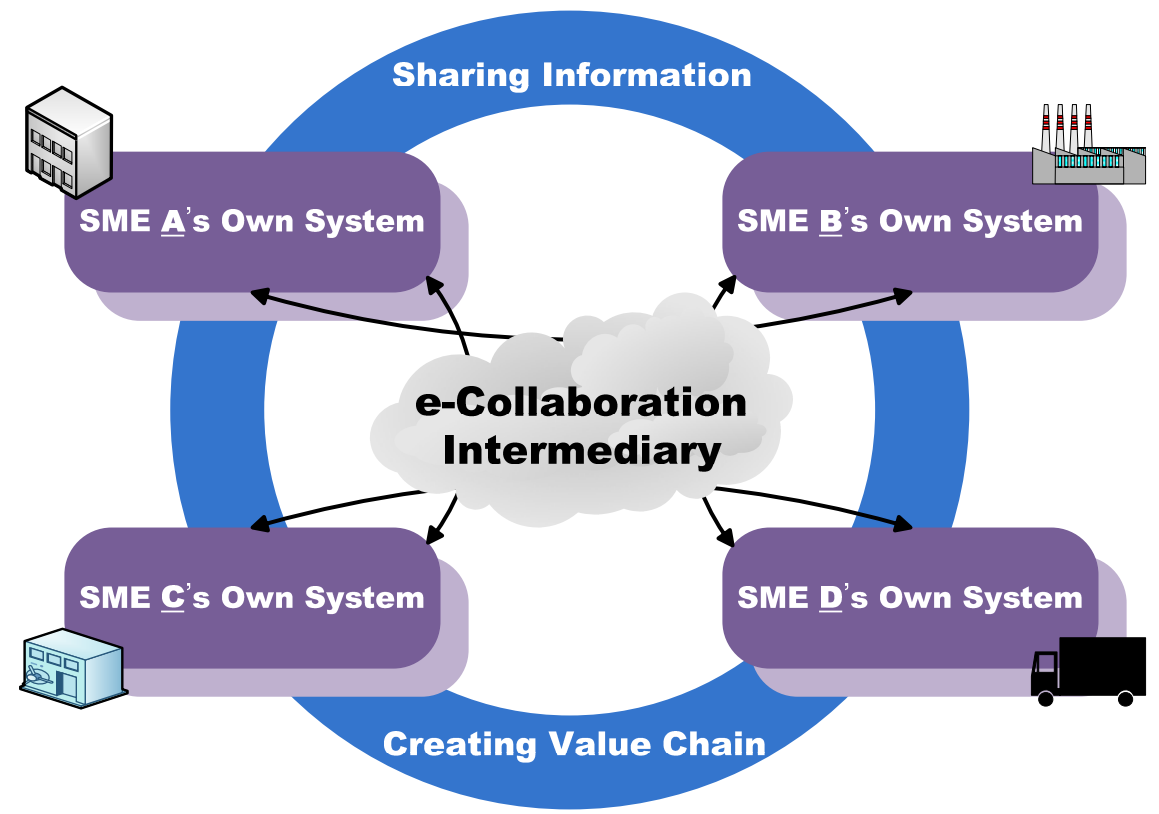

Figure 1 Desirable e-Collaboration structure

As shown in the above figure, as four SMEs intend to establish the collaboration, the e-Collaboration Intermediary is defined to support this collaborative environment. In other words, the e-Collaboration Intermediary is in charge of supporting that SMEs with the different system each other can form the collaborative business relationship while they use their own unique systems.

\section{APPLICABLE E-COLLABOATION SYSTEM ARCHITECTURE}

In the previous chapter, the paper defined the horizontal collaborative system as the desirable collaboration, and suggested the participation of the e-Collaboration 
Intermediary for the structure to realize the collaboration in between SMEs.

\section{The basic technology}

Even SMEs have set up the individual systems regardless of their sizes. But these systems can be the factors which limit the information sharing necessary for the eCollaboration. Accordingly, the technological foundation is required for the easy access and integrated management. There are Registry/Repository, Web Service, and Mapping in the basic technologies which can constitute the technological foundation of the Intermediary.

\section{- Registry/Repository}

As the space for the information storage, the repository includes not only the simple information on data, but also business strategies, business models, specifications of design, etc. Especially, as one combined storage space to connect the heterogeneous model types, it is the basic technology to store and manage all the knowledge as a whole which the enterprises, which constitute the collaboration, have. It can be understood as compatibility, and it is required to adapt the repository in order to flexibly deal with the information of each enterprise which has the huge data. Moreover, as the technology of 'registry \& repository' is included in ebXML Working Group, the importance of utilizing it is getting bigger. Assuming that the information for the collaboration in between the enterprises is treated in the registry \& repository as the informatized space, it can be explained that the actual data for the co-sharing is to be stored in the repository, and the registry can store and mange the meta-data which can refer to the information stored in repository (Hefner, 2000).

\section{- Web Service}

The Web Service is the foundation which can make a series of business activities possible by combining the single or plural numbers of applications through standardization of them using the internet. Especially, by ruling the definition on the standard of data which the enterprises give and take each other, it takes the role as a connecting link to quite flexibly overcome the difference of communication in between the mutual heterogeneous operating systems or program languages.

As it can be the distributed architecture, the web service can be used while not influencing the specific application which constitutes the systems of the individual 
enterprises, and the compatibility is secured only by modifying the application directly related to the system of own company with no need to modify the whole system. This function of the web service makes the efficient combination possible with faster and less expensive cost than substituting the hardware or applying the combined software in order to combine the application in between the enterprises (Vinoski, 2003).

\section{- Mapping}

As the systems of the individual enterprises are different each other, it is necessary to connect the different data or business process for the compatibility of each different system. This concept can be defined by the mapping. According to the methods, it can be divided into the 'Direct Code Mapping' and the 'Basic Code Mapping'.

As for the direct code mapping, it is required to make out the codes one by one in order to match the data of the individual enterprises in one to one. Accordingly, it takes a lot of cost for time and money for the mapping, and in case of a new enterprise's participation in the collaboration, it has the inefficient aspect as the big investment should follow up.

On the contrary, the basic code mapping is the conversion method setting the basis; it sets up the basic codes, and makes the individual systems follow them. The data and process of the various systems which are converted into the basic codes can secure the compatibility by giving and taking the information each other based upon the basic codes. Also, by adapting the basic code mapping, it is possible to reduce the cost of time and money for the mapping, and it has the virtues that the new enterprise can efficiently and flexibly collaborate with all the existing enterprises only by following the basic codes in case of a new enterprise's participation in the existing collaboration.

\section{Architecture}

Figure 2 shows the system architecture which this paper suggests based upon the basic technology and concept of information and communication. Aiming at the horizontal e-Collaboration, this system architecture focuses on creating the new business models and processes by maintaining the existing system of the individual enterprises through securing the compatibility in between the various systems. 


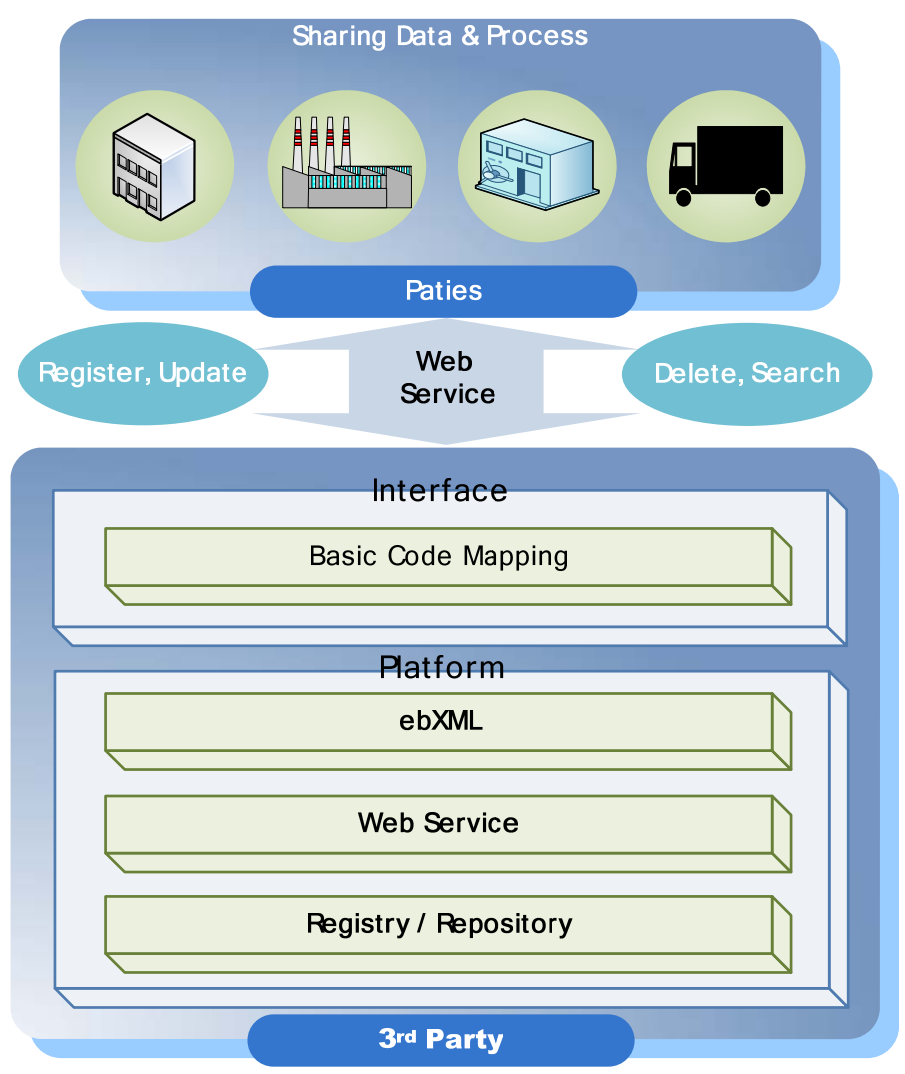

Figure 2 System Architecture for e-Collaboration

The e-Collaboration Platform, which has been defined to be developed and operated by the collaboration intermediary, is the core of the architecture taking not only the role of the interface to make the data or process compatible through 'Basic Code Mapping', but the role of registering and managing data or process of 'Registry/ Repository' through 'Web Service'. Let's take a closer look at this.

Assuming a series of the e-collaboration processes such as the procurement, manufacture, distribution, and sales etc., as the informatized space, 'registry/repository' stores and manages the information on the product, procurement, order, sales, settlement, transportation, stockpiles etc., which is necessary to be shared for the collaboration, and also guarantees that the individual enterprises can access to this information systematically.

From the detailed perspective, all the information is stored in the repository, but the information summary and retrieval is done through the registry. Only in order for the 
information shared in the informatized space to be provided to the individual enterprises, it should be converted to the information form fitting each enterprise. And the different protocols should be simplified for the compatibility of the business process. Accordingly, applying the basic code mapping, it is possible to naturally proceed with the process by storing and managing the information within the e-Collaboration Platform.

At the same time, in order to use the service provided by the collaboration system, it should be guaranteed that the enterprises can access to the e-Collaboration Platform in which the actual process of information is carried out. Therefore, the web service is to be applied as the method for the information exchange in between the enterprises. By applying the web service which is the standardized software technology to combine the different kinds of computer programs, the information sharing based upon the internet is possible. Namely, it is possible to deliver the information on the company to the partner without any delay, and on the contrary, it is possible to check all the information on the partner which exists in the e-Collaboration Platform through the web service. Especially, as the business activities by the collaboration are exercised through the web service in semblance, it is more appropriate for SMEs which lack the expertise.

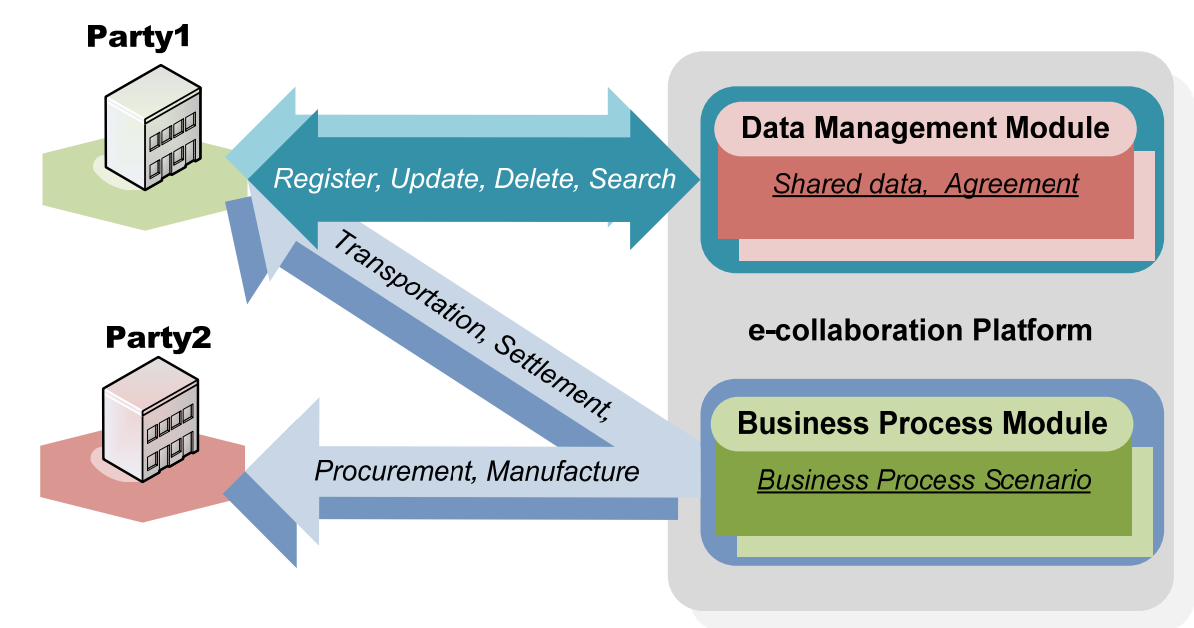

Figure 3 Information Processing of e-Collaboration Platform

On the other side, regarding the information processing structure of the eCollaboration Platform based upon the architecture proposed previously, it is judged that Figure 3 is more desirable. First, it is necessary to make out the scenario for the business process in order to carry out the business process in between SMEs smoothly, and to 
make the business process as libraries in the Business Process Module by each stage according to the scenario. For instance, it is required to constitute the transactions so that it can be possible to provide the request or the response document for the procurement, manufacture, transportation, or sales, and to support that the business activity can be carried out just like in the one enterprise. Especially, as the business process scenario gets in library, it has the virtues to enlarge the size of the collaboration by only making out newly additional transaction on case by case basis.

As all the data necessary for the e-Collaboration is stored in the Data Management Module, it makes the search, modification, and removal of information possible in between SMEs. The data can be divided into hierarchical data and none-hierarchical data, and especially, the former should be comprehensively librarized for coexistence of various and different hierarchy; which is because the data having the hierarchical structure such as the raw material classification, product classification, and stock classification etc. has the possibility of the information loss during the mapping owing to each SME's hierarchical criteria while the mapping is easy to be done for nonehierarchical data such as the product unit and currency unit.

In conclusion, the e-Collaboration Platform is to process the information by the Business Process Module and the Data Management Module. The Data Management Module manages the business information, product information, and information on the transaction agreement, and the Business Process Module operates actual process systematically. Like this way, it enhances the efficiency of the modules to carry out the similar functions belonging in the same category in the one module, which can improve the capability of information processing of the e-Collaboration Platform.

\section{Aspect of Technology}

\section{EXPECTED EFFECT}

As for the system architecture suggested in the paper for the e-Collaboration Intermediary, its application can be justified in its open, horizontal, and result-oriented characteristics. The superiority in the open characteristics can be found in the utilization of the web service based on the internet; it is because it can reduce the burden for establishing the system environment accompanying the participation of a new enterprise in the collaboration to apply the web service, and also its application is quite easy. Also, together with the web service, it has the distributed structure which enables the enterprise to participate in the collaboration with the unique system of its own, by adapting basic 
code mapping. Through this, the open characteristics can be expected by lowering entry barrier to the collaboration.

In addition, the distributed structure can be free from the logic of power because any participants are not subordinate to any enterprises' standards by making the business processes and sharing the information equally. This indicates horizontal characteristics of system architecture of e-Collaboration Intermediary.

The result-oriented characteristics are expected as well. It is possible to establish more uniform and sophisticated strategies as the enterprises participating in the collaboration can exercise their responsibility with the same purpose of the continuous circulation of the business process. Moreover, it can positively influence the result with the strong motive accompanying the collaboration as the benefits of individual enterprises through this e-collaborative architecture is fairer than any other structure.

\section{Aspect of Business}

The collaboration did not begin theoretically, but was originated through the demand for the work-site operations and the enterprises relationship. Accordingly, it is necessary to evaluate the business effect under technological environment (Narayanan \& Raman, 2004).

The theoretical and empirical study on the expected effect of the intermediary as the concept to connect business process of each enterprise or newly provide the eCollaboration process has been scarcely done yet. Only the studies on the reason of existence, function, and role of the intermediary (Peng \& Ilinitch, 1998; Peng et al., 2000; Trabold, 2002; Choi, 2004) have been done from the perspective of making the business activities efficient or creating the new business. The e-Collaboration Intermediary suggested in the paper promotes the development of SMEs by creating and supporting the business models and processes as the same function and role; in this regard, the paper aims at studying on the expected effect of business on the prolongation of the previous studies.

First of all, in the aspect that the e-Collaboration deals with the transactional structures in between the enterprises, the reason of existence, function, and role of the intermediary can be found in the paradigm of Transaction Cost Theory by Williamson. Transaction Cost Theory is to try to find the optimized transactional structure to minimize the cost, and it means that the market activity evolves toward the direction to reduce the cost(Williamson, O.E., 1979). From this viewpoint, the following effect can be 
expected as the functional and procedural aspect in the utilization of the intermediary.

First, the intermediary will be the form which can establish the platform to support various systems and commercialize it by using the superior position based upon the excellent expertise and objectivity compared to SMEs. Accordingly, it is possible to reduce the costs of time and money necessary for developing and operating the collaborative models or processes through the e-Collaboration intermediary by the enterprises themselves. Also, as the service by the intermediary can be provided in packets and units through the ICT and internet, it will be possible to reduce the unnecessary burdens of the enterprises by charging as much costs as they used (Hagel III \& Brown, 2001).

Second, it can actively meet the demands of SMEs on the state-of-the-art technologies through the simplified method. The service of the intermediary will be provided in packets just like the popular informatization services, and the update of the collaborative system will be easy as well. Therefore, SMEs using the intermediary can optimize the collaborative environment through the inexpensive and simple procedure as they can be provided with the state-of-the-art technologies through the regular updates. Also, as it is possible to be additionally provided with the package only to the relevant solution in case of the demands of SMEs on the new services, SMEs can promptly respond to the structural changes of the collaboration, and the inefficiency caused by the unnecessary function can be prevented owing to the possibility of the selective adaptation.

Like this way, it can be emphasized that the e-Collaboration Intermediary should be adapted in the aspect of functional and procedural cost reduction, which is suggested in Transaction Cost Theory.

On the other hand, assuming that the intermediary itself shall bring about the change and innovation in the industry and service, the role to get rid of the psychological entry barrier to this movement should be necessary.

For instance, in order to respond to all the possible problems in the initial stage of horizontal collaboration which lacks the manpower, information and experience, the enterprises need the intermediary role which can promote organizing the market by providing the professional and objective function of system supporting to all the participating enterprises. This can be understood as the structural support of the intermediary based upon 'Theory of Economic Development' by Schumpeter, and the following effect can be expected(Schumpeter, 1934). 
First, regarding developing the system for the collaboration, the term of collaboration can work as the dangerous factor, and the intermediary can be expected to reduce this risk. In case of constituting the collaboration through the intermediary with the platform for the collaborative system, it will be possible to receive the solution as soon as possible with no obstacles in the short-term collaboration as well as in the longterm one. In other words, the effect to induce the organization of collaboration is confirmed by enhancing the transactional credibility and raising the possibility for the development of more sophisticated function through the objective and professional third party.

Second, in case of being provided with the customized service from the intermediary, the enterprises can reduce the burden for the liquidation resulted from failure because they can reduce the cost and risk for entering the new business; which means the role of intermediary as the collaboration promoter which can reduce restraints and diversify the models for the collaborative business in the end, with regard to the creation or enlargement of the new business through the collaboration.

From the perspective of the 'Theory of Economic Development', the adaptation of the e-Collaboration Intermediary can be emphasized as the leverage role to organize the collaborative environment and to make the collaboration form by lowering entry barrier.

\section{CONCLUSION}

It is judged that the adaptation of the e-Collaboration Intermediary suggested in the paper in order to promote the collaboration in between SMEs has the considerable possibility in the technological and business perspective. From the technological perspective, the system architecture of the e-Collaboration Intermediary can be helpful to maximize the size and benefits of the collaboration because it has the open, horizontal, and result-oriented structure. From the business perspective, the e-Collaboration Intermediary can be expected to promote the collaboration by reducing the cost and lowering the entry barrier to the collaboration. So it is expected that this paper can actually help the revitalization of SMEs which invisibly contribute the economy worldwide. And we think that introduction of e-marketplace which adapts our ecollaboration architecture could make a new business model; if this e-MP is introduced, many candidate, SMEs which want play a role in each e-collaboration process(i.e. procurement, manufacture, distribution, and sales etc.) will take part in the e-MP. And 
then optimal process will be formed by selected SMEs.

On the other hand, in order to be the substantial workable mechanism, the eCollaboration Intermediary should complete its role as the partner to adjust the individual conditions of SMEs, and it needs to sophisticate its function based upon the standpoints of SMEs; It is because the consultocracy, which distorts the market, can be caused while the standpoints of SMEs, the users are excluded if focused on providing the service based upon the e-Collaboration Intermediary, supplier.

\section{REFERENCES}

Anthony, T. (2000). Supply Chain Collaboration; Success in the New Internet Economy. Achieving Supply Chain Excellence Through Technology, 2, 241-244.

Bernroider, E. and Koch, S. (2000) Differences in Characteristics of the ERP System Selection Process between Small or Medium Sized and Large Organizations. Long Beach,California: Proceedings of the Sixth Americas Conference of Information Systems.

Chen, L.; Haney, S.; Pandzik, A.; Spigarelli, J. \& Jesseman, C. (2003). Small Business Internet Commerce; A Case Study. Information Resources Management Journal, 16(3), 17-41.

Choi, S. H.; Chang, S. G. \& Shin, M.S. (2005). Business Governance and Digital Convergence; Case Study in Telecommunications, Finance, and Broadcasting Industries. Information and Society, 8(1), 17-38.

Choi. Y. R. (2004). An Empirical Study on the Motivation of Global e-Trade Intermediary in Korea. e-Business Studies, 5(2), 57-73.

Hagel III, J. \& Brown, J.S. (2001). Your Next IT Strategy. Harvard Business Review, November 2001, 105-113.

Hefner, R. (2000). Managing Projects through a Corporate Repository. IEEE Computer Society. $33^{\text {rd }}$ Hawaii International Conference on System Sciences, 7, 7051.

Konings, J. \& Roodhooft, F. (2002). The Effect of E-Business on Corporate Performance; Firm Level Evidence for Belgium. De Economist, 150(5), 569-581.

Narayanan, V.G. \& Raman, A. (2004). Aligning Incentives in Supply Chains. Harvard Business Review, November 2004, 94-102.

Nie, J. (2007). A Study of Information Technology Adoption for Small and Medium Sized Enterprises Strategic Competitiveness. IEEE Computer Society. 3rd Wireless Communications, Networking and Mobile Computing, 4342-4346. 
Nøkkentved, C. \& Hedaa, L. (2000). Collaborative Processes in E-Supply Networks. Bath, U.K. : Proceedings of the 16th International Marketing and Purchasing Group Conference.

Park, J. \& Yang, J. (2005). A Case Study on e-Collaboration of Federal Structure. Journal of Society for e-Business Studies, 10(5), 35-52.

Peng, M. W., Hill, C. W. L. \& Wang, D. Y. L. (2000). Schumpeterian Dynamics Versus Williamsonian Considerations; A Test of Export Intermediary Performance. Journal of Management Studies, 37(2), 167-184.

Peng, M. W. \& Ilinitch, A. Y. (1998). Export Intermediary Firms: A Note on Export Development Research. Journal of International Business Studies, 29(33), 609-620.

Porter, M. E. (2001). Strategy and the Internet. Harvard Business Review, March 2001, 62-79.

Pratt, J. H. (2002). E-Biz: strategies for small business success. U.S.: US SBA Office of Advocacy.

Rao, S. S., Metts, G \& Mora Monge, C.A. (2003). Electronic Commerce Development in Small and Medium Sized Enterprises; A Stage Model and Its Implications. Business Process Management Journal, 9(1), 11-32.

Salo, J. \& Karjaluoto, H. (2006). IT_Enabled Supply Chain Management. Contemporary Management Research, 2(1), 17-30.

Sawhney, M. \& Mandal, S. (2000). Marketing the Web World Wide ; A Roadmap Globalization of E-Commerce. Working Paper, February, Kellogg Graduate School of Management.

Schumpeter, J. A.(1934). The Theory of Economic Development: An Inquiry into Profits, Capital, Credit, Interest, and the business cycle. (1949 ed.). Cambridge, MA: Harvard University Press.

Steinfield, C. (2002). Understanding Click and Mortar e-Commerce Approach; A Conceptual framework and Research Agenda. Journal of Interactive Advertising, 2(2). (available at http://www.jiad.org/article19)

Teng, J., Cheon, M. \& Grover, V. (1995). Decisions to Outsource Information Systems Functions; Testing a Strategy-theoretic Discrepancy Model. Decision Sciences, 26(1), 75-103.

Trabold, H. (2002). Export Intermediation; An Empirical Test of Peng and Ilinitch. Journal of International Business Studies, 33(2), 327-344.

Vinoski, S. (2003). Integration with Web Services. IEEE Internet Computing, Nov. 
Dec., $75-77$.

Watson, G. (2004). Uncertainty and Contractual Hazard in the Film Industry; Managing Adversarial Collaboration with Dominant Suppliers. Supply Chain Management: An International Journal, 9(5), 402-409.

Williamson, O.E. (1979). Transaction-cost Economics ; The Governance of Contractual Relations. Journal of Law and Economics, 22, 233-262. 